\title{
REDUCTION OF SLOW-FAST PERIODIC SYSTEMS WITH APPLICATIONS TO POPULATION DYNAMICS MODELS
}

\author{
M. MARVÁ \\ Departamento de Matemáticas, Universidad de Alcalá, \\ 28871 Alcalá de Henares, Spain \\ marcos.marva@uah.es \\ J.-C. POGGIALE \\ U.M.R. C.N.R.S., 7249-MIO, Institut Pytheas \\ Campus de Luminy 13288, Marseille Cedex 9, France \\ jean-christophe.poggiale@univ-amu.fr \\ R. BRAVO DE LA PARRA \\ Departamento de Matemáticas, Universidad de Alcalá, \\ 28871 Alcalá de Henares, Spain \\ rafael.bravo@uah.es \\ Received 5 April 2011 \\ Revised 17 October 2011 \\ Published 28 May 2012 \\ Communicated by O. Diekmann
}

This work deals with the approximate reduction of a nonautonomous two time scales ordinary differential equations system with periodic fast dynamics. We illustrate this technique with the analysis of two models belonging to different fields in ecology. On the one hand, we deal with a two patches periodic predator-prey model with a refuge for prey. Considering migrations between patches to be faster than local interaction allows us to study a three-dimensional system by means of a two-dimensional one. On the other hand, a two time scales periodic eco-epidemic model is addressed by considering two competing species, one of them being affected by a periodic SIR epidemic process which is faster than inter-species interactions. The difference between time scales allows us to study the asymptotic behavior of the four-dimensional system by means of a planar, reduced one. Furthermore, we propose a methodology straightforwardly applicable to a very large class of two time scales periodic systems.

Keywords: Slow-fast systems; periodic systems; population dynamics; eco-epidemic models.

AMS Subject Classification: 34N05 


\section{Introduction}

The description of ecological systems in terms of mathematical models makes those latter to be complex and thus requiring some reduction to be analytically tractable. This complexity arises from the fact that a detailed model necessarily includes different observations and processes, each of them related to a specific scale. A simplification of this situation needs to translate model processes from one scale to another one by transferring information between them, what it is called scaling. Hierarchy theory provides the conceptual framework of how processes and components of an ecological system inter-relate and how they can be ordered, see Refs. 22 and 26. Considering models coupling two different processes always comes along with an assumption concerning the time scales corresponding with each process. In other words, the question is: do these processes evolve on different time scales? And, if so, how does the difference between the time scales affect the behavior of the complete system?

In mathematical terms a system including several interacting organization levels can be seen as a system with different time scales. Each organization level consists of interacting entities with their own dynamics. Those entities belonging to a given level with strong or fast interactions can be grouped giving rise to the entities at next level. Mathematically the process of up-scaling consists in deriving global variables and their dynamics from the lower level based on the existence of different time scales. Roughly, this is done by considering those events occurring at the fastest scale as being instantaneous with respect to the slower ones, which entails a reduction of the number of variables and parameters needed to describe the evolution of the system at the upper level.

An example of this general framework is the so-called aggregation methods which study the relationship between a large class of two time scales complex systems and their corresponding aggregated or reduced ones. A review on these methods in different mathematical settings with updated bibliography can be found in Refs. 3 and 4. Aggregation techniques are particularly well developed for autonomous ordinary differential equations, the Fenichel center manifold theorems ${ }^{13}$ and the geometric singular perturbation theory ${ }^{28,29}$ being their mathematical basis. In short, an autonomous system of ordinary differential equations with two time scales can be expressed in the following form:

$$
\frac{d n}{d \tau}=f(n)+\varepsilon s(n)
$$

with $n \in \mathbb{R}^{m}$, where maps $f$ and $s$ represent the fast and slow dynamics, respectively, and $\varepsilon$ is the small positive parameter measuring the time scales ratio when it is possible. To perform its approximate aggregation, system (1.1) is firstly converted into slow-fast form by means of an appropriate change of variables 
$n \in \mathbb{R}^{m} \rightarrow(x, y) \in \mathbb{R}^{m-k} \times \mathbb{R}^{k}:$

$$
\left\{\begin{array}{l}
\frac{d x}{d \tau}=F(x, y)+\varepsilon \bar{S}(x, y), \\
\frac{d y}{d \tau}=\varepsilon S(x, y),
\end{array}\right.
$$

where $x$ represents the fast variables and $y$ the slow variables. Finding the transformation $n \mapsto(x, y)$ which yields the slow-fast form (1.2) of system (1.1) could be a difficult task and the construction of general algorithms solving this problem is presently an active research line. On the other hand, in some applications, as we will see later, the context gives a natural way to define the so-called global variables $y$ and thus to express system (1.1) in slow-fast form.

The reduction process now consists in taking $\varepsilon=0$ in the first equation of the slow-fast form $(1.2), d x / d \tau=F(x, y)$, and assuming, for constant $y$, that there exists asymptotically stable equilibrium $x^{*}(y)$, in building up an aggregated system for the global variables with the following form:

$$
\frac{d y}{d t}=S\left(x^{*}(y), y\right)
$$

where $t=\varepsilon \tau$ represents the slow time variable. Under certain hypotheses the asymptotic behavior of system (1.1) can be studied through system (1.3).

The purpose of this work is extending these reduction techniques to systems of nonautonomous ordinary differential equations. In various situations, these systems represent more realistic population models compared with autonomous ones due to the flexibility to include time-varying features of the environment (light, temperature, relative humidity or resources availability) as well as demographic characteristics of the involved populations (migrations or reproduction) which are usually subjected to daily or seasonal variations. A particular and very relevant case of nonautonomous system is the periodic one, which is frequently found as model of natural systems.

We develop in Sec. 2 the approximate reduction of a general class of two time scales systems of periodic ordinary differential equations of the form:

$$
\varepsilon \frac{d n}{d t}=f(t, n)+\varepsilon s(t, n) .
$$

To our knowledge the only result of approximate aggregation of a nonautonomous system is found in Ref. 25. In this work, the fast dynamics is considered nonautonomous and assumed to tend to a stationary periodic solutions depending on global variables; averaging techniques together with the aforementioned Fenichel center manifold theorems allow to proceed to the reduction of the system. There is no overlap with our results since we introduce periodic time-dependent fast dynamics together with nonautonomous slow dynamics and we use Hoppensteadt theorems on singular perturbations Ref. 19 to justify the suggested reduction. 
In Sec. 3 we illustrate the aggregation techniques developed in Sec. 2 applying them to a two patches prey-predator model where prey can migrate between the patch where predators are and a refuge. These terms describing predator and prey behavior are considered to be periodic and, furthermore, local predator-prey interactions are described according to the functional response presented in Ref. 30 (now with periodic coefficients). Prey migrations are assumed to be fast when compared with predator-prey interactions. We obtain the reduced system and study with its help the asymptotic behavior of solutions of the initial system. Section 4 contains another application to eco-epidemics model and, finally, Sec. 5 is devoted to outline further perspectives and applications of these techniques presented herein. For the convenience of the reader we have included a brief summary of Hoppensteadt results in Appendix A as well as some technical results in Appendix B and C.

\section{Reduction Theorem}

In this section we present the reduction of the following class of nonautonomous two time scale systems:

$$
\varepsilon \frac{d n}{d t}=f(t, n)+\varepsilon s(t, n),
$$

with $n \in \mathbb{R}^{m}$ and where $f$ and $s$ represent the fast and slow dynamics, respectively.

This kind of systems with $f$ and $s$ not depending on $t$ have been extensively studied and applied to different biological models using approximate aggregation methods, see Refs. 3 and 4 for recent reviews, which first step is to transform the system into slow-fast form by means of an appropriate change of variables. To reproduce this step with the nonautonomous system (2.1) we assume that it exists a change of variables $n \in \mathbb{R}^{m} \rightarrow(x, y) \in \mathbb{R}^{m-k} \times \mathbb{R}^{k}$ that yields the following system:

$$
\left\{\begin{array}{l}
\varepsilon \frac{d x}{d t}=F(t, x, y)+\varepsilon \bar{S}(t, x, y) \\
\frac{d y}{d t}=S(t, x, y)
\end{array}\right.
$$

where $x$ and $y$ stand for the fast and the slow variables, respectively. Though it might be difficult to find the appropriate transformation leading to the slow-fast form (2.2) of system (2.1), in some applications, like the prey-predator model (3.2) in next section, it is straightforwardly done. The search for the slow variables, those ones kept constant by fast dynamics, yields the key of the transformation. In system (3.2) the obvious candidates for slow variables are the total number of prey and predators, which do not change through fast dynamics (prey movements between patches) and so they evolve at the slow time scale.

The autonomous case of the slow-fast system (2.2) is reduced by means of Fenichel center manifold theorems. ${ }^{3,4}$ The asymptotic behavior of the complete initial system is then studied with the help of a reduced system for the global 
variables called aggregated system. Here, for the nonautonomous case, we notice that system (2.2) is a particular case of system (A.1) in Appendix A taking

$$
f(t, x, y, \varepsilon)=F(t, x, y)+\varepsilon \bar{S}(t, x, y) \quad \text { and } \quad g(t, x, y, \varepsilon)=S(t, x, y),
$$

so, provided the hypotheses hold, we can apply the Hoppensteadt results summarized in Theorem A.1. System (A.4), that in this case takes the form

$$
\frac{d \bar{y}}{d t}=S(t, \Phi(t, \bar{y}), \bar{y}), \quad \text { with } \Phi(t, y) \text { satisfying } F(t, \Phi(t, y), y)=0
$$

plays a similar role to the aggregated system in the autonomous case, in the sense that some features of its asymptotic behavior can be translated in terms of system (2.2) via Theorem A.1.

We next present a particular version of Theorem A.1 for system (2.2) where we consider $F$ to be periodic. The use of this reduction result is illustrated in the following sections.

Theorem 2.1. Let us consider system (2.2) with $F$ periodic on $t$ and let $\Omega=$ $\left[t_{0}, \infty\right) \times K_{R} \times K_{R^{\prime}}$, where $K_{R}=\left\{x \in \mathbb{R}_{+}^{m-k}:|x| \leq R\right\}$ and $K_{R^{\prime}}=\left\{y \in \mathbb{R}_{+}^{k}:|y| \leq\right.$ $\left.R^{\prime}\right\}$, satisfying:

(1) Functions $F, S$ and $\bar{S}$ are $\mathcal{C}^{2}(\Omega)$ and any solution of the system (2.2) beginning in $K_{R} \times K_{R^{\prime}}$ remains there for $t \in\left[t_{0}, \infty\right)$.

(2) There is a function $\Phi(t, y) \in \mathcal{C}^{2}\left(\left[t_{0}, \infty\right) \times B_{R^{\prime}}\right)$ such that for any $(t, y) \in$ $\left[t_{0}, \infty\right) \times K_{R^{\prime}}$ the following hold:

(a) $F(t, \Phi(t, y), y)=0$.

(b) The real part of the eigenvalues of $J_{x} F(t, \Phi(t, y), y)$ is negative.

(3) The system of equations

$$
\frac{d \bar{y}}{d t}=S(t, \Phi(t, \bar{y}), \bar{y})
$$

has a solution for $t_{0} \leq t<\infty\left(\right.$ say, $\left.y^{*}(t)\right)$ that it is uniformly asymptotically stable.

If $y_{0}$ is in the domain of attraction of $y^{*}(t)$ and $x_{0}$ is in the domain of attraction of the equilibrium $\Phi\left(t_{0}, y_{0}\right)$ for system $\frac{d \bar{x}}{d t}=F\left(t_{0}, \bar{x}, y_{0}\right)$, then the solution $(x(t), y(t))$ of system (2.2) with $x\left(t_{0}\right)=x_{0}$ and $y\left(t_{0}\right)=y_{0}$ and the solution of system (2.3) with $\bar{y}\left(t_{0}\right)=y_{0}$ satisfy

$$
x(t)=\Phi(t, \bar{y}(t))+o(1), \quad y(t)=\bar{y}(t)+o(1),
$$

as $\varepsilon \rightarrow 0^{+}$uniformly on any interval of the form $t_{0}<t_{1} \leq t<\infty$.

Proof. The only point that we need to justify is that condition (2b) implies Hypothesis H3 of Appendix A, the rest of conditions being mere translations of 
Hypotheses H1, H2 and H4 to the present case. So, we need to prove that equilib$\operatorname{rium} \Phi(\alpha, \beta), \alpha \in\left[t_{0}, \infty\right)$ and $\beta \in K_{R^{\prime}}$, of system

$$
\frac{d X}{d t}=F(\alpha, X, \beta)
$$

is asymptotically stable uniformly in $(\alpha, \beta)$. The fact that $F$ is periodic in its first argument, $\alpha$, implies that we only need to prove the result for $(\alpha, \beta)$ in the compact set $\mathcal{K}=\left[t_{0}, t_{0}+\omega\right] \times K_{R^{\prime}}$ where $\omega$ is the period of $F$.

Let us consider $\varphi(t, \alpha, \beta)$, any solution of system $(2.4)$, we define $z(t)=$ $\varphi(t, \alpha, \beta)-\Phi(\alpha, \beta)$ and linearize around $\Phi(\alpha, \beta)$ to get that

$$
\frac{d z}{d t}=A(\alpha, \beta) z+f(\alpha, z, \beta)
$$

where $A(\alpha, \beta)=J_{x} F(\alpha, \Phi(\alpha, \beta), \beta)$ and $f(\alpha, z, \beta)=o(|z|)$ uniformly in $(\alpha, \beta) \in \mathcal{K}$, that is, for any $\varepsilon>0$ there is $\delta>0$ such that

$$
|f(\alpha, z, \beta)| \leq \varepsilon|z|,
$$

for $|z|<\delta$.

The fact that all eigenvalues of $A(\alpha, \beta)$ have negative real part with $(\alpha, \beta)$ taking values in the compact set $\mathcal{K}$ implies that there exist two positive constants $\gamma$ and $M$ such that for every $t \geq 0$ and every $(\alpha, \beta) \in \mathcal{K}$

$$
\left|e^{t A(\alpha, \beta)}\right| \leq M e^{-\gamma t}
$$

The variation of constants formula applied to system (2.5) yields

$$
z(t)=e^{\left(t-t_{0}\right) A(\alpha, \beta)} z\left(t_{0}\right)+\int_{t_{0}}^{t} e^{(t-s) A(\alpha, \beta)} f(\alpha, z(s), \beta) d s .
$$

Let us choose $\varepsilon$ such that $\varepsilon M<\gamma$ and let $\delta$ be chosen so that (2.6) is satisfied, we have

$$
|z(t)| \leq M e^{-\gamma\left(t-t_{0}\right)}\left|z\left(t_{0}\right)\right|+\int_{t_{0}}^{t} \varepsilon M e^{-\gamma(t-s)}|z(s)| d s, \quad t \geq t_{0} .
$$

Setting $g(t)=e^{\gamma t}|z(t)|$ this inequality implies

$$
g(t) \leq M g\left(t_{0}\right)+\int_{t_{0}}^{t} \varepsilon M g(s) d s, \quad t \geq t_{0} .
$$

An application of Gronwall's inequality yields

$$
g(t) \leq M e^{\varepsilon M\left(t-t_{0}\right)} g\left(t_{0}\right), \quad t \geq t_{0},
$$

which in turn implies

$$
|z(t)| \leq M e^{-(\gamma-\varepsilon M)\left(t-t_{0}\right)}\left|z\left(t_{0}\right)\right|, \quad t \geq t_{0}
$$


for all values of $t \geq t_{0}$ for which $|z(t)|<\delta$. Since $\gamma-\varepsilon M>0$, this inequality implies $|z(t)|<\delta$ for all $t \geq t_{0}$ as long as $\left|z\left(t_{0}\right)\right|<\frac{\delta}{M}$. Consequently (2.7) holds for all $t \geq t_{0}$ provided $\left|z\left(t_{0}\right)\right|<\frac{\delta}{M}$, which implies the uniform asymptotic stability we needed to prove.

\section{Periodic Predator-Prey Model with Fast Migrations}

In this section we present a two time scales predator-prey model including a refuge for prey. Using the method developed in Sec. 2 a reduced model is derived and studied. Finally, from Theorem 2.1 we obtain some results on the behavior of solutions of the initial model.

We consider a predator-prey community inhabiting a heterogeneous habitat. We simulate heterogeneity by considering a patchy environment and letting individuals migrate between patches. For the sake of simplicity we consider a two patches environments, one of them being (say, the first one) a predator-free region, i.e. a refuge for prey. Prey population at patch $i=1,2$ is noted by $n_{i}$ and $p$ stands for predator population at the second region. Prey migrate from patch $i$ at a constant rate $m_{i}$. Migrations are understood as individual movements between different zones of the habitat, so that we consider migrations to be faster than local dynamics. Demographic processes and predator-prey interactions are supposed to be periodic functions of time with the same period $T$. In the first patch, the refuge, prey evolve under a logistic law with net growth rate $r_{1}(t)$ and carrying capacity $K_{1}(t)$. In the second patch, the interaction zone, predator-prey dynamics are described as follows: Logistic growth for prey in absence of predators, $r_{2}(t)$ and $K_{2}(t)$ being the net growth rate and the carrying capacity respectively, predator exponential decay in absence of prey, with mortality rate $\mu(t)$, and the following functional response:

$$
\frac{\varphi(t) n_{2}}{1+\gamma(t) p}
$$

This functional response type was first used in Ref. 30 (although the model considered therein is autonomous). It is inspired on the observation that in Nature, prey and predator are not always interacting. Far from these, any group of prey is at any moment in different "vulnerability states" with respect to predator, depending on spatial position (e.g. in hiding places) and activity (e.g. resting versus actively feeding). These states can be simplified into "vulnerable" and "invulnerable". Furthermore, the authors assumed that changing the vulnerability state is a faster process than predator-prey interactions. Then, a mechanistic-type argument gives rise to the functional response (3.1) (see the Appendix section in Ref. 30 for a detailed explanation). From a different point of view, the functional response (3.1) can be thought to describe predator interference, being a particular case of the well-known Beddington ${ }^{7}$ DeAngelis ${ }^{11}$ predator-prey model.

Clearly, the existence of a refuge is linked with the "invulnerable state". Besides, prey migrations between the refuge and the interaction arena are related with the change of vulnerability state. Thus, using the functional response given by (3.1) 
is fully compatible (and suitable) with our settings. The proposed model is represented by the following system of nonautonomous ordinary differential equations with periodic coefficients:

$$
\left\{\begin{array}{l}
\varepsilon \frac{d n_{1}}{d t}=-m_{1} n_{1}+m_{2} n_{2}+\varepsilon r_{1}(t) n_{1}\left(1-\frac{n_{1}}{K_{1}(t)}\right) \\
\varepsilon \frac{d n_{2}}{d t}=m_{1} n_{1}-m_{2} n_{2}+\varepsilon\left(r_{2}(t) n_{2}\left(1-\frac{n_{2}}{K_{2}(t)}\right)-\frac{\varphi(t) n_{2}}{1+p} p\right) \\
\frac{d p}{d t}=-\mu(t) p+\frac{\bar{\varphi}(t) n_{2}}{1+p} p
\end{array}\right.
$$

where the functions $r_{1}, r_{2}, K_{1}, K_{2}, \mu, \varphi$ and $\bar{\varphi}$ are positive, bounded away from zero, periodic with the same period $T$ and $\mathcal{C}^{2}(\mathbb{R})$. The small positive parameter $\varepsilon$ represents the ratio of time scales. Periodic variations are considered to occur on the slow time scale (say, yearly) meaning the following. We do not expect large variations in these parameters describing population dynamics in the short term (e.g. within a week), but these large variations are allowed in the long term (e.g. after several months).

\subsection{Reduction of the system}

Though system (3.2) seems to be in slow-fast form (2.2), setting $\varepsilon=0$, the manifold of equilibria for the fast dynamics does not verify condition (2c) of Theorem 2.1 because the Jacobian is the constant matrix $\left(\begin{array}{rr}-m_{1} & m_{2} \\ m_{1} & -m_{2}\end{array}\right)$ that has an eigenvalue equal to 0 . This happens because it is hidden another slow variable: the total number of preys that we note

$$
n=n_{1}+n_{2}
$$

To obtain the appropriate slow-fast form (2.2) of system (3.2) we change variables $\left(n_{1}, n_{2}, p\right)$ into $\left(n_{1}, n, p\right)$ in system $(3.2)$, which yields

$$
\left\{\begin{array}{l}
\varepsilon \frac{d n_{1}}{d t}=m_{2} n-\left(m_{1}+m_{2}\right) n_{1}+\varepsilon r_{1}(t) n_{1}\left(1-\frac{n_{1}}{K_{1}(t)}\right) \\
\frac{d n}{d t}=r_{1}(t) n_{1}\left(1-\frac{n_{1}}{K_{1}(t)}\right)+r_{2}(t)\left(n-n_{1}\right)\left(1-\frac{n-n_{1}}{K_{2}(t)}\right)-\frac{\varphi(t)\left(n-n_{1}\right)}{1+p} p \\
\frac{d p}{d t}=-\mu(t) p+\frac{\bar{\varphi}(t)\left(n-n_{1}\right)}{1+p} p .
\end{array}\right.
$$

To prove that system (3.4) verifies condition (1) of Theorem 2.1, as the regularity assumption obviously holds, we justify that it exists and appropriate positively invariant set. In system (3.2) it is straightforward to see that the positive octant is 
positively invariant and so the same is true for $\left\{\left(n_{1}, n, p\right) \in \mathbb{R}_{+}^{3}: n_{1} \leq n\right\}$ in system (3.4). Now, from the second equation of system (3.4) we have that

$$
\frac{d n}{d t} \leq r_{1}(t) n_{1}\left(1-\frac{n_{1}}{K_{1}(t)}\right)+r_{2}(t)\left(n-n_{1}\right)\left(1-\frac{n-n_{1}}{K_{2}(t)}\right)
$$

and calculating the maximum of the second term as a function of $n_{1} \in[0, n]$ we can find a constant $M_{n}$ such that, for every $n \geq M_{n}, \frac{d n}{d t}<0$ in $n_{1} \in[0, n]$ and for any $p \geq 0$. Finally, from the third equation of system (3.4) we can obtain a constant $M_{p}$ such that $\frac{d p}{d t}<0$ for every $p \geq M_{p}$ and $n_{1} \leq n \leq M_{n}$. Thus, the set $\left\{\left(n_{1}, n, p\right) \in \mathbb{R}_{+}^{3}: n_{1} \leq n \leq M_{n}, p \leq M_{p}\right\}$ is positively invariant for system (3.4).

To obtain function $\Phi$ of condition (2) of Theorem 2.1, i.e. the fast equilibrium, we need to solve for $n_{1}$ equation

$$
m_{2} n-\left(m_{1}+m_{2}\right) n_{1}=0
$$

and, thus, we define

$$
\Phi(t, n, p)=\frac{m_{2}}{m_{1}+m_{2}} n=\nu^{*} n .
$$

It is straightforward to prove that $\Phi(t, n, p)$ verifies condition (2b) of Theorem 2.1 and that the equilibrium $\Phi\left(t_{0}, n_{0}, p_{0}\right)$ is globally asymptotically stable for equation

$$
\frac{d \bar{n}_{1}}{d \bar{t}}=m_{2} n_{0}-\left(m_{1}+m_{2}\right) \bar{n}_{1} .
$$

The reduced system (2.3) has in this case the following form:

$$
\left\{\begin{array}{l}
\frac{d \bar{n}}{d t}=(a(t)-b(t) \bar{n}) \bar{n}-\frac{c(t) \bar{n}}{1+\bar{p}} \bar{p}, \\
\frac{d \bar{p}}{d t}=-\mu(t) \bar{p}+\frac{\bar{c}(t) \bar{n}}{1+\bar{p}} \bar{p},
\end{array}\right.
$$

where

$$
\begin{aligned}
& a(t)=r_{1}(t) \nu^{*}+r_{2}(t)\left(1-\nu^{*}\right), \quad b(t)=\frac{r_{1}(t)\left(\nu^{*}\right)^{2}}{K_{1}(t)}+\frac{r_{2}(t)\left(1-\nu^{*}\right)^{2}}{K_{2}(t)}, \\
& c(t)=\varphi(t)\left(1-\nu^{*}\right) \quad \text { and } \quad \bar{c}(t)=\bar{\varphi}(t)\left(1-\nu^{*}\right) .
\end{aligned}
$$

Now Theorem 2.1 applies to system (3.2) provided we can find a uniformly asymptotically stable solution of system (3.6) as stated in next theorem.

Theorem 3.1. Let $\left(n_{1}(t), n_{2}(t), p(t)\right)$ be the solution of the initial value problem

$$
\begin{cases}\varepsilon \frac{d n_{1}}{d t}=-m_{1} n_{1}+m_{2} n_{2}+\varepsilon r_{1}(t) n_{1}\left(1-\frac{n_{1}}{K_{1}(t)}\right), & n_{1}\left(t_{0}\right)=n_{1}^{0}, \\ \varepsilon \frac{d n_{2}}{d t}=m_{1} n_{1}-m_{2} n_{2}+\varepsilon\left(r_{2}(t) n_{2}\left(1-\frac{n_{2}}{K_{2}(t)}\right)-\frac{\varphi(t) n_{2}}{1+p} p\right), & n_{2}\left(t_{0}\right)=n_{2}^{0}, \\ \frac{d p}{d t}=-\mu(t) p+\frac{\bar{\varphi}(t) n_{2}}{1+p} p, & p\left(t_{0}\right)=p^{0} .\end{cases}
$$


If the reduced system (3.6) has a solution for $t_{0} \leq t<\infty$ that it is uniformly asymptotically stable with $\left(n_{1}^{0}+n_{2}^{0}, p_{0}\right)$ in its domain of attraction and $(\bar{n}(t), \bar{p}(t))$ is the solution of $(3.6)$ for $\left(\bar{n}\left(t_{0}\right), \bar{p}\left(t_{0}\right)\right)=\left(n_{1}^{0}+n_{2}^{0}, p^{0}\right)$ then

$$
n_{1}(t)=\nu^{*} \bar{n}(t)+o(1), \quad n_{2}(t)=\left(1-\nu^{*}\right) \bar{n}(t)+o(1), \quad p(t)=\bar{p}(t)+o(1),
$$

as $\varepsilon \rightarrow 0^{+}$uniformly on any interval of the form $t_{0}<t_{1} \leq t<\infty$.

\subsection{Analysis of the reduced system}

In this section we provide with sufficient conditions for the existence and the stability of periodic solutions of system (3.6) entailing predator-prey coexistence or predator extinction. Straightforward calculations reveal that system (3.6) has no equilibrium points, so that we seek for $T$-periodic solution. From now on, we note' for $\frac{d}{d t}$.

For further purposes, we recall that the coefficients of the reduced system are periodic, positive and bounded away from zero. Thus, they achieve strictly positive minimum and maximum, which we note as

$$
\begin{gathered}
a_{L} \leq a(t) \leq a_{M}, \quad b_{L} \leq b(t) \leq b_{M}, \quad c_{L} \leq c(t) \leq c_{M}, \\
\mu_{L} \leq \mu(t) \leq \mu_{M}, \quad \bar{c}_{L} \leq \bar{c}(t) \leq \bar{c}_{M} .
\end{gathered}
$$

We recall also the following known results, which will be used in the proof of Proposition 3.3.

\section{Proposition 3.1. Let}

$$
z^{\prime}=h(t, z)
$$

be a T-periodic system on $t$, where $h \in \mathcal{C}^{1}\left(\mathbb{R} \times \mathbb{R}^{m}\right)$. The existence of a convex bounded compact positively invariant region $\mathcal{R} \subset \mathbb{R}^{m}$ for system (3.9) implies the existence of a periodic solution for system (3.9).

Proof. Let us note $z\left(t, t_{0}, z_{0}\right)$ the solution system (3.9) such that $z\left(t_{0}\right)=z_{0}$ and consider the Poincaré operator $\psi_{T}: \mathcal{R} \rightarrow \mathcal{R}$ defined by

$$
\psi_{T}\left(z_{0}\right)=z\left(t_{0}+T, t_{0}, z_{0}\right),
$$

which maps each initial value $z_{0} \in \mathcal{R}$ on the region $\mathcal{R}$ into the value at time $t=t_{0}+T$ of the solution of problem (3.6) which starts at $z_{0}$ in $t=t_{0}$. This is a continuous map and $\mathcal{R}$ is convex and by the Brouwer's fixed-point theorem ${ }^{9}$ this operator has a fixed point, which means that there exists a solution $z^{*}\left(t, t_{0}, z_{0}^{*}\right)$ of the problem (3.6) such that

$$
z^{*}\left(t_{0}, t_{0}, z_{0}^{*}\right)=z^{*}\left(t_{0}+T, t_{0}, z_{0}^{*}\right) .
$$

Then $z^{*}\left(t, t_{0}, z_{0}^{*}\right)$ is a periodic solution due to the uniqueness of solutions of system (3.9). 
In case of the reduced system (2.3) being $T$-periodic on $t$ and having found a $T$-periodic solution $y^{*}(t)$ of this system, the uniformly asymptotic stability of $y^{*}(t)$ can be established using Floquet's theory.

Proposition 3.2. Assume that the system $z^{\prime}=h(t, z)$ is T-periodic on $t$ and let $z^{*}(t)$ be an T-periodic solution. Consider also the linearized system around $z^{*}$

$$
z^{\prime}=h_{z}\left(t, z^{*}(t)\right) z
$$

Then, any of the following conditions assures that $z^{*}(t)$ is uniformly asymptotically stable:

(1) The characteristic multipliers of system (3.10) have modulus less than one.

(2) The zero solution of system (3.10) is uniformly asymptotically stable and the linear system (3.10) has no periodic solutions different from the zero solution.

Proof. It follows from the considerations done in Sec. 4.2 in Ref. 12 (in particular, Theorem 4.2.1).

Proposition 3.3. If condition

$$
0<\frac{\mu_{M}}{\bar{c}_{L}}<\frac{a_{L}-c_{M}}{b_{M}}
$$

holds, then there exists a positive periodic solution $\left(n^{*}(t), p^{*}(t)\right)$ of system (3.6) which is uniformly asymptotically stable.

Proof. The proof is accomplished in several steps: first we find a convex invariant region $\mathcal{R}$ for system (3.6). Applying Proposition 3.1 we get the existence of at least one positive periodic solution for system (3.6) within $\mathcal{R}$. Then, we linearize system (3.6) around such a positive periodic solution to prove that it is uniformly asymptotically stable.

Step 1: Existence of a positive periodic solution. Let us assume that $n \cdot p \neq 0$. Using bounds (3.8) and system (3.6) it is straightforward that

$$
\left\{\begin{array}{l}
a_{L}-b_{M} n-\frac{c_{M} p}{1+p} \leq n^{\prime} \leq a_{M}-b_{L} n-\frac{c_{M} p}{1+p} \\
-\mu_{M}+\frac{\bar{c}_{L} n}{1+p} \leq n^{\prime} \leq-\mu_{L}+\frac{\bar{c}_{M} n}{1+p}
\end{array}\right.
$$

Using (3.12), direct calculations yield curves

$$
\begin{gathered}
n_{+}^{\prime}(n):=\frac{a_{L}-b_{M} n}{c_{M}-a_{L}+b_{M} n}, \quad n_{-}^{\prime}(n):=\frac{a_{M}-b_{L} n}{c_{L}-a_{M}+b_{L} n}, \\
p_{-}^{\prime}(n):=\frac{\bar{c}_{M}}{\mu_{L}} n-1, \quad p_{+}^{\prime}(n):=\frac{\bar{c}_{L}}{\mu_{M}} n-1,
\end{gathered}
$$



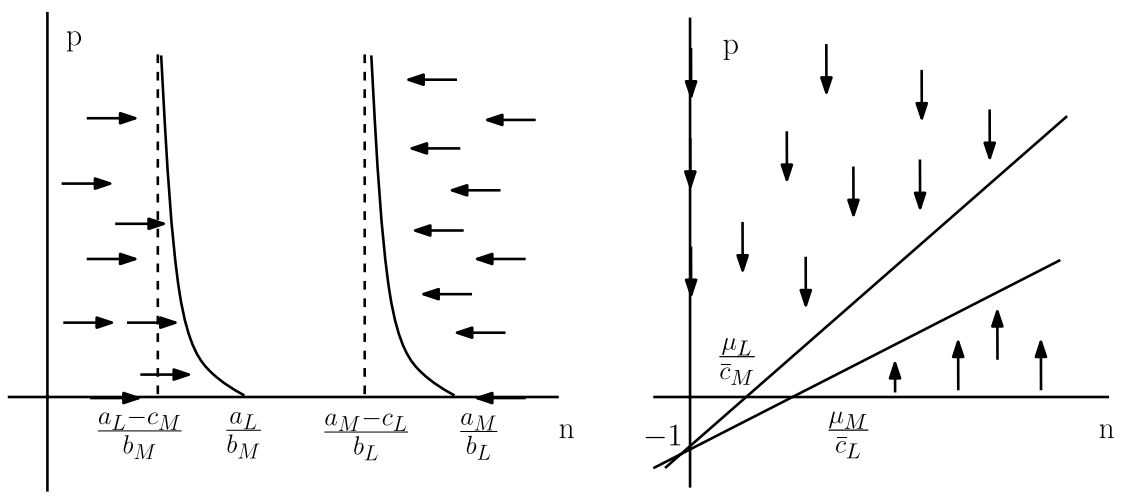

Fig. 1. Left and right: regions where the sign of $n^{\prime}$ and $p^{\prime}$ is constant. The curves are noted, from left to right, $n_{+}^{\prime}(n), n_{-}^{\prime}(n), p_{-}^{\prime}(n)$ and $p_{+}^{\prime}(n)$.

delimiting regions of the first quadrant where the sign of $n^{\prime}$ and $p^{\prime}$ are constant, as shown in Fig. 1.

Depending on the relative position of the nulclines (3.13) we can find different scenarios. We seek for a positively invariant, convex region $\mathcal{R}$ bounded away from the axes. $\mathcal{R}$ is a rectangular region. Thus, we shall find $r_{i} \in \mathbb{R}, i=1, \ldots, 4$ such that $\mathcal{R}:=\left[r_{1}, r_{2}\right] \times\left[r_{3}, r_{4}\right]$. Keeping in mind the configuration of the nulclines, vertex $\left(r_{1}, r_{3}\right)$ is defined by the intersection of curves $n=\frac{\left(a_{L}-c_{M}\right)}{b_{M}}$ and $p_{+}^{\prime}(n)$. Besides, it is needed $r_{2}>\frac{a_{M}}{b_{L}}$ and $r_{4}$ is given by the intersection of curves $n=r_{2}$ and $p_{-}^{\prime}(n)$. From the bounds for the derivatives of $(n(t), p(t))$ given by Eq. (3.12), the comparison theorem and the construction of the rectangle, it follows that $\mathcal{R}=\left[r_{1}, r_{2}\right] \times\left[r_{3}, r_{4}\right]$ is the region we were looking for. Figure 2 shows the rectangular closed invariant

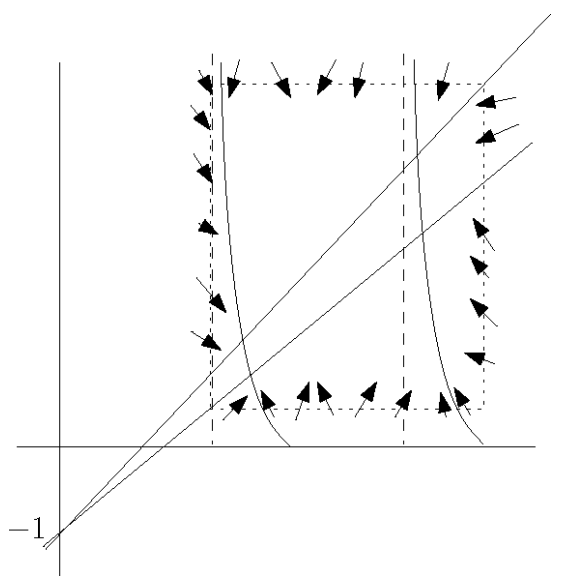

Fig. 2. The invariant region $\mathcal{R}$. 
region $\mathcal{R}$ and from Proposition 3.1 we get the existence of a positive $T$-periodic solution $\left(n^{*}(t), p^{*}(t)\right)$ for system (3.6).

Step 2: The periodic solution is uniformly asymptotically stable. In order to assure the attraction of the periodic solution, according to Proposition 3.2 we study the stability of the zero solution of the variational problem of (3.6) at $\left(n^{*}(t), p^{*}(t)\right)$ (i.e. we linearize the problem at the periodic solution, see Ref. 12). Thus, we deal with the system

$$
X^{\prime}=A(t) X
$$

where

$$
A(t)=\left(\begin{array}{cc}
a(t)-2 b(t) n^{*}(t)-\frac{c(t) p^{*}(t)}{1+p^{*}(t)} & \frac{-c(t) n^{*}(t)}{\left(1+p^{*}(t)\right)^{2}} \\
\frac{\bar{c}(t) p^{*}(t)}{1+p^{*}(t)} & -\mu(t)+\frac{\bar{c}(t) n^{*}(t)}{\left(1+p^{*}(t)\right)^{2}}
\end{array}\right)
$$

$X=\left(x_{1}, x_{2}\right)^{T}$ (super-index $T$ stands for transposition) and $n^{*}(t), p^{*}(t)$ are the components of the periodic solution. Keeping in mind the fact that

$$
\left\{\begin{array}{l}
\frac{n^{*^{\prime}}(t)}{n^{*}(t)}=a(t)-b(t) n^{*}(t)-\frac{c(t) p^{*}(t)}{1+p^{*}(t)} \\
\frac{p^{*^{\prime}}(t)}{p^{*}(t)}=-\mu(t)+\frac{\bar{c}(t) n^{*}(t)}{1+p^{*}(t)}
\end{array}\right.
$$

the change of variables $y_{1}=\frac{x_{1}}{n^{*}}, y_{2}=\frac{x_{2}}{p^{*}}$ transforms the system (3.14) into

$$
Y^{\prime}=B(t) Y
$$

where $Y=\left(y_{1}, y_{2}\right)^{T}$ and

$$
B(t)=\left(b_{i j}(t)\right)=\left(\begin{array}{cc}
-b(t) n^{*}(t) & \frac{-c(t) p^{*}(t)}{\left(1+p^{*}(t)\right)^{2}} \\
\frac{\bar{c}(t) n^{*}(t)}{1+p^{*}(t)} & \frac{-\bar{c}(t) p^{*}(t) n^{*}(t)}{\left(1+p^{*}(t)\right)^{2}}
\end{array}\right)
$$

which is equivalent to (3.14). Applying Appendix B.1 finishes the proof.

Proposition 3.4. If condition (3.11) holds, then there exists a unique T-periodic positive solution of problem (3.6) within region $\mathcal{R}$.

Proof. It follows from an application of the topological degree and the details can be found in Appendix C.

In fact, the positive periodic solution whose existence is guaranteed by Proposition 3.3 is a global attractor. The proof of this result is related with the stability 
of the semi-trivial solution of system (3.6). Namely, if we let $p(t)=0$, then system (3.6) simplifies in

$$
n^{\prime}=(a(t)-b(t) n) n, \quad n\left(t_{0}\right)=n_{0},
$$

which can be explicitly solved and was studied, for instance, in Ref. 14. In this paper, it was shown that if $a(t)>0$ and $b(t)>0$ are periodic functions with common period $T$ then there exists a unique positive periodic solution $n_{0}^{*}(t)$ for (3.17) which is globally asymptotically stable and such that

$$
\frac{a_{L}}{b_{M}} \leq n_{0}^{*}(t) \leq \frac{a_{M}}{b_{L}}
$$

where $a_{L}, a_{M}, b_{L}, b_{M}$ stand for the maximum and the minimum of functions $a(t)$ and $b(t)$. We refer to $\left(n_{0}^{*}(t), 0\right)$ as the semi-trivial solution of system (3.6). Besides, both positive semi-axes are invariant sets for system (3.6). The following results concern the stability of the semi-trivial solution of the reduced system.

Proposition 3.5. Let $\left(n_{0}^{*}(t), 0\right)$ be the semi-trivial solution of the reduced system (3.6) and

$$
a_{1}:=\int_{t_{0}}^{t_{0}+T}\left(-\mu(t)+\bar{c}(t) n_{0}^{*}(t)\right) d t
$$

Then

(1) If $a_{1}<0$ then, $\left(n_{0}^{*}(t), 0\right)$ is uniformly asymptotically stable.

(2) If $a_{1}>0$ then, $\left(n_{0}^{*}(t), 0\right)$ is unstable.

Proof. Linearizing the reduced system (3.6) around the semi-trivial solution yields

$$
\left(\begin{array}{l}
x_{1}^{\prime} \\
x_{2}^{\prime}
\end{array}\right)=\left(\begin{array}{cc}
a(t)-2 b(t) n_{0}^{*}(t) & -c(t) n_{0}^{*}(t) \\
0 & -\mu(t)+\bar{c}(t) n_{0}^{*}(t)
\end{array}\right)\left(\begin{array}{l}
x_{1} \\
x_{2}
\end{array}\right) .
$$

This is a linear periodic system and we need to calculate the Floquet exponents in order to study its stability. System (3.20) is an upper-triangular one and can be explicitly solved. The second equation in (3.20) is

$$
x_{2}^{\prime}=\left(-\mu(t)+\bar{c}(t) n_{0}^{*}(t)\right) x_{2}
$$

and its solution is given by

$$
x_{2}(t)=x_{2}\left(t_{0}\right) \exp \left(\int_{t_{0}}^{t}-\mu(s)+\bar{c}(s) n_{0}^{*}(s) d s\right) .
$$

Replacing this expression into the first equation and solving it we get a fundamental system:

$$
\Phi(t)=\left(\begin{array}{cc}
\exp \left(\int_{t_{0}}^{t}\left(a(s)-2 b(s) n_{0}^{*}(s)\right) d s\right) & \Phi_{12}(t) \\
0 & \exp \left(\int_{t_{0}}^{t}-\mu(s)+\bar{c}(s) n_{0}^{*}(s) d s\right)
\end{array}\right)
$$


where $\Phi_{12}(t)$ is a complex expression. Moreover, the Floquet exponents are given by

$$
\begin{aligned}
& \lambda_{1}=\exp \left(\int_{t_{0}}^{t_{0}+T}\left(a(s)-2 b(s) n_{0}^{*}(s)\right) d s\right), \\
& \lambda_{2}=\exp \left(\int_{t_{0}}^{t_{0}+T}-\mu(s)+\bar{c}(s) n_{0}^{*}(s) d s\right) .
\end{aligned}
$$

On the one hand, $\left|\lambda_{2}\right|<1$ because of condition (3.19). On the other hand, $\left|\lambda_{1}\right|<1$ because $b(s) n_{0}^{*}(s)>0$ and

$$
\int_{t_{0}}^{t_{0}+T}\left(a(s)-b(s) n_{0}^{*}(s)\right) d s=0 .
$$

Corollary 3.1. The semi-trivial solution $\left(n_{0}^{*}(t), 0\right)$ is a global attractor for the solutions of system (3.6) if

$$
\frac{a_{M}}{b_{L}}<\frac{\mu_{L}}{\bar{c}_{M}}
$$

Proof. It follows from the proof of Proposition 3.5 that condition (3.21) implies $a_{0}<0$, i.e. the semi-trivial solution $\left(n_{0}^{*}(t), 0\right)$ is uniformly asymptotically stable. Thanks to (3.18), there exists $\varepsilon_{0}>0$ such that for each $0<\varepsilon<\varepsilon_{0}$ every solution of (3.6) with initial values within

$$
\mathcal{W}_{\varepsilon}:=\left[\frac{a_{L}}{b_{M}}-\varepsilon, \frac{a_{M}}{b_{L}}+\varepsilon\right] \times[0, \varepsilon]
$$

is attracted by the semi-trivial solution. Let $\rho$ be a positive constant such that $0<\rho<\min \left\{\varepsilon, \frac{\left(a_{L}-c_{M}\right)}{b_{M}}\right\}$ and $n_{+, \rho}^{\prime}(n)=n_{+}^{\prime}(n)-\rho$. For a fixed $\varepsilon$, we note the region bounded by the curves $n_{+, \rho}^{\prime}(n)$ and $n=\frac{a_{M}}{b_{L}}$ by $\mathcal{Q}$ (including those points on the curves). Moreover, we define

$$
\begin{gathered}
\mathcal{Q}_{2}:=\mathcal{Q} \cup \mathcal{W}_{\varepsilon}, \\
\mathcal{Q}_{1}=\left\{(n, p) \in \mathbb{R}^{2} ; 0<n<\frac{a_{L}}{b_{M}} ; 0<p\right\} \backslash \mathcal{Q}_{2}, \\
\mathcal{Q}_{3}=\left\{(n, p) \in \mathbb{R}^{2} ; \frac{a_{M}}{b_{L}}<n ; 0<p\right\} \backslash \mathcal{W}_{\varepsilon} .
\end{gathered}
$$

As $\frac{\mu_{L}}{\bar{c}_{M}} \geq \frac{a_{M}}{b_{L}}$, from Eq. (3.12) we notice that there exist positive constants $\delta_{1}$ and $\delta_{3}$ such that $n^{\prime}(t)<-\delta_{3}<0$ in $\mathcal{Q}_{3}$ and $n^{\prime}(t)>\delta_{1}>0$ in $\mathcal{Q}_{1}$. Thus, solutions starting in both regions $\mathcal{Q}_{1}$ and $\mathcal{Q}_{3}$ will leave them (and so, reach $\mathcal{Q}_{2}$ and stay in) after a transient time. The same reason implies that solutions starting within $\mathcal{Q}_{2}$ will remain in $\mathcal{Q}_{2}$. Moreover, there exists $\delta_{2}>0$ such that $p^{\prime}(t)<-\delta_{2}<0$ in $\mathcal{Q}_{2}$. Thus, every positive solution $(n(t), p(t))$ in $\mathcal{Q}_{2}$ is strictly decreasing and

$$
\lim _{t \rightarrow+\infty}(n(t), p(t)) \in \mathcal{W}_{\varepsilon},
$$

which finishes the proof. 
Corollary 3.2. If condition (3.11) holds, then the positive periodic solution whose existence is guaranteed by Proposition 3.3 is a global attractor to system (3.6).

Proof. The proof is similar to that of Corollary 3.1 .

\subsection{Analysis of the complete model}

In this section we analyze the complete system (3.2) by means of Theorem 3.1 along with these results achieved for the reduced system (3.6) in the previous section. Conditions (3.11) and (3.21) are sufficient conditions entailing predator-prey coexistence or predator extinction. Nevertheless, these conditions do not cover all the possible cases, and numerical simulations illustrate the uncovered cases. First, let us summarize all the previous results in the following theorem.

Theorem 3.2. Let $\nu^{*}$ and $n_{0}^{*}(t)$ be the fast equilibrium (3.5) and the periodic solution of Eq. (3.17), respectively. Consider $\left(n_{1}(t), n_{2}(t), p(t)\right)$ the solution of the initial value problem (3.7) with initial values $\left(n_{1}^{0}, n_{2}^{0}, p_{0}\right) \in \mathbb{R}_{+}^{3}$ and $(\bar{n}(t), \bar{p}(t))$ the solution of the reduced system (3.6) starting at $\left(n_{1}^{0}+n_{2}^{0}, p_{0}\right)$. Then, if any of conditions (3.11) or (3.21) hold,

$$
n_{1}(t)=\nu^{*} \bar{n}(t)+o(1), \quad n_{2}(t)=\left(1-\nu^{*}\right) \bar{n}(t)+o(1), \quad p(t)=\bar{p}(t)+o(1),
$$

as $\varepsilon \rightarrow 0^{+}$uniformly on any interval of the form $t_{0}<t_{1} \leq t<\infty$. We recall that:

(1) If condition (3.11) holds, then

$$
\lim _{t \rightarrow \infty}(\bar{n}(t), \bar{p}(t))=\left(n^{*}(t), p^{*}(t)\right)
$$

uniformly, where $\left(n^{*}(t), p^{*}(t)\right)$ is the periodic positive solution of Proposition 3.3.

(2) If condition (3.21) holds, then

$$
\lim _{t \rightarrow \infty}(\bar{n}(t), \bar{p}(t))=\left(n_{0}^{*}(t), 0\right)
$$

uniformly.

Proof. It is a direct consequence of all the previous analysis.

Now, we turn our attention to the uncovered cases, namely, we consider that

$$
\frac{a_{L}-c_{M}}{b_{M}}<\frac{\mu_{M}}{\bar{c}_{L}} \text { and } \quad \frac{\mu_{L}}{\bar{c}_{M}}<\frac{a_{M}}{b_{L}} .
$$

This case cannot be analyzed analytically. Numerical experiments show that, within this case, we can have either a positive solution (coexistence) or a semi-trivial omega limit (predators exclusion) for system (3.2). Let us illustrate this fact through the following numerical simulations.

Case 1: Coexistence. We consider a set of parameter (see Fig. 3) which leads to condition (3.22). For these parameters, we represent, on the one hand, the state 

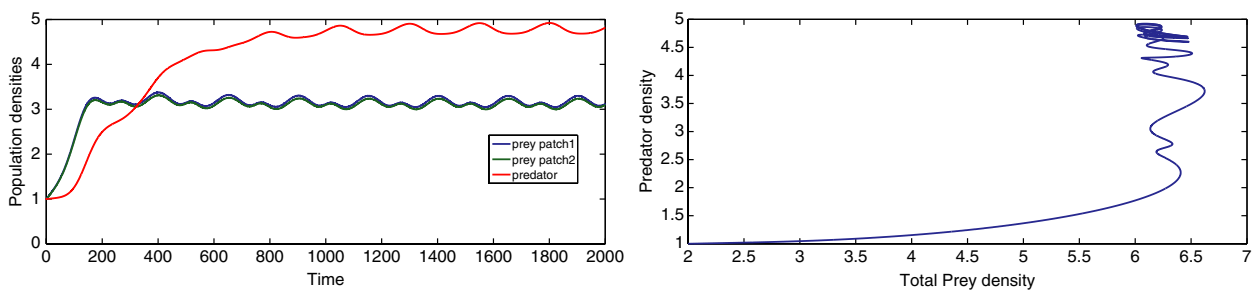

Fig. 3. Left: state variables versus time: a positive periodic orbit exists. Right: phase portrait of the reduced model illustrating the positive periodic orbit. Parameter values: $m_{1}=1, m_{2}=1$, $r_{1 L}=1, r_{2 L}=0.1, r_{1 M}=3, r_{2 M}=2.1, \phi_{L}=0.1, \bar{\phi}_{L}=0.8 * \phi_{L}, \phi_{M}=2.1, \bar{\phi}_{M}=\phi_{M} * 0.8$, $\mu_{L}=0.01, \mu_{M}=1.01, T=5, \varepsilon=0.02, K_{1 L}=5, K_{2 L}=1, K_{1 M}=9, K_{2 M}=5$.
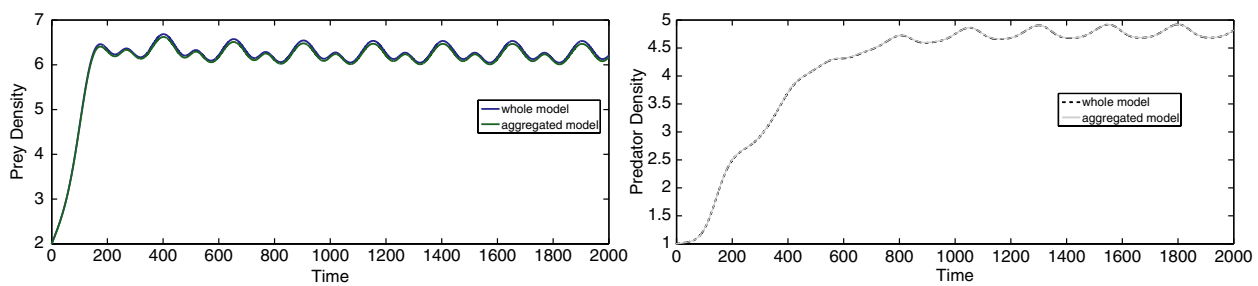

Fig. 4. Comparison of the total prey density (left) and total predator density (right) simulated with the full and the reduced model. Parameter values are these stated in Fig. 3.

variables versus time showing that a positive periodic orbit exists and, on the other hand, a phase portrait illustrating the positive periodic orbit (see Fig. 3). In addition we have included a comparison of the total prey/predator density simulated with the full and the reduced model (see Fig. 4).

Let us assume that there exists a positive solution for the reduced system (3.6) for the parameter values listed in Fig. 3. We can go through Step 2 in the proof of Proposition 3.3 to ensure that, in fact, every positive periodic solution is uniformly asymptotically stable.

Case 2: Predators exclusion. We consider now the parameter set values in Fig. 5, which fulfill condition (3.22). Again, for these parameter values, Fig. 5 represents the state variables as function of time and the corresponding phase portrait, showing that the predator can be excluded. In this case, we could not establish analytically the stability of the semi-trivial. Nevertheless, the simulation in Fig. 6 shows that results obtained with the parameter values stated in Fig. 5 for the general and reduced system are coherent.

\subsection{Summary of results}

As a result of the previous analysis, we have set threshold quantities for predatorprey coexistence and predator extinction in terms of the relative shape of certain "vital parameters" of the reduced system, namely, $a(t), b(t)$ and $\mu(t), \bar{c}(t)$.

In a non-spatially distributed system, $\frac{a(t)}{b(t)}$ stands for the carrying capacity of the corresponding ecosystem, in the sense of (3.18) (and comments made there). 

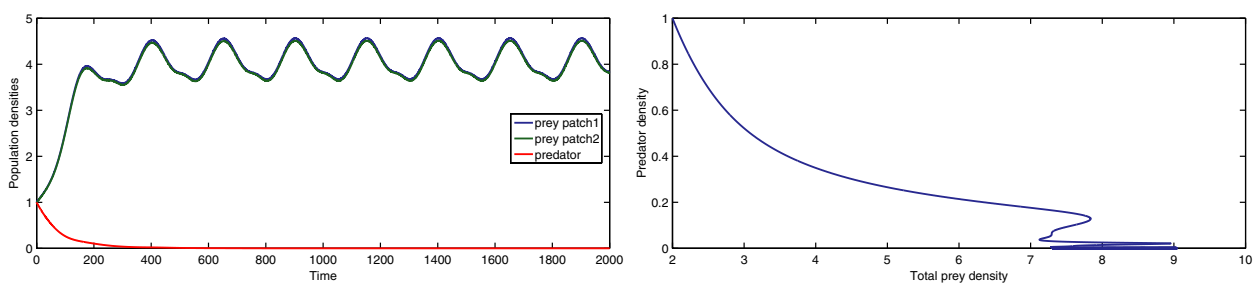

Fig. 5. Left: state variables versus time: prey permanence; predators extinction. Right: phase portrait of the reduced model illustrating predators exclusion. Parameter values: $m_{1}=1, m_{2}=1$, $r_{1 L}=1, r_{2 L}=0.1, r_{1 M}=3, r_{2 M}=2.1, \phi_{L}=0.1, \bar{\phi}_{L}=0.2 * \phi_{L}, \phi_{M}=2.1, \bar{\phi}_{M}=0.2 * \phi_{M}$, $\mu_{L}=0.6, \mu_{M}=1.6, T=5, \varepsilon=0.02, K_{1 L}=5, K_{2 L}=1, K_{1 M}=9, K_{2 M}=5$.
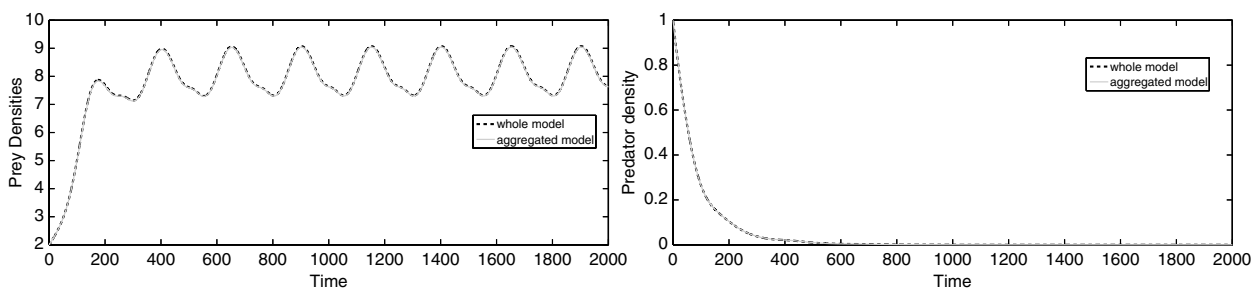

Fig. 6. Comparison of the total prey density (left) and total predator density (right) simulated with the full and the reduced model. Parameter values are these stated in Fig. 5.

According to the definition of the coefficients of the reduced system (3.6), it follows that

$$
\frac{a(t)}{b(t)}=\frac{\left(r_{1}(t) \nu_{1}^{*}+r_{2}(t)\left(1-\nu_{1}^{*}\right)\right) K_{1}(t) K_{2}(t)}{r_{1}(t)\left(\nu_{1}^{*}\right)^{2} K_{2}(t)+r_{2}(t)\left(\left(1-\nu_{1}^{*}\right)\right)^{2} K_{1}(t)},
$$

which is the carrying capacity for the spatially distributed prey population when we consider fast migrations and periodic coefficients at each region.

On the other hand, $\frac{\mu(t)}{\bar{c}(t)}$ stands for the ratio between predator's mortality rate and benefits of captures for predators.

Thus, we have stated condition (3.11) ensuring the existence of a coexistence state and condition (3.21) implying the exclusion of predators in terms of the coefficients of the system. Summing up:

- There exists an attracting periodic coexistence state if $\frac{\mu_{M}}{\bar{c}_{L}}<\frac{a_{L}-c_{M}}{b_{M}}$.

- Predators die out at low population densities when $\frac{a_{M}}{b_{L}}<\frac{\mu_{L}}{\bar{c}_{M}}$.

- There exists a range of intermediate cases (see condition (3.22)) which are indefinite, meaning that both predators exclusion or coexistence can arise.

In the context of the system we are dealing with, coefficients $a(t), b(t)$ and $\bar{c}(t)$ depend on $\nu_{1}^{*}$, which is related with prey migrations. In fact, from Corollary 3.1 
and the definition of the coefficients (3.8), small changes in $\nu_{1}^{*}$ may entail a change in the stability of the semi-trivial solution of the reduced system and thus, induce the extinction of predators.

\section{Further Applications: Eco-Epidemics Models}

In this section we briefly describe another example of a nonautonomous two time scales model. Now, fast dynamics are considered also to be time-dependent.

Eco-epidemiology is a research area that studies the interactions between community and epidemic processes. Despite its youth, it is rapidly becoming a field of study in its own rights. Anderson and $\mathrm{May}^{2}$ were the first to consider a predatorprey model where prey species was infected by some disease. Since then, many authors have proposed and studied different predator-prey models in the presence of disease, mainly affecting preys ${ }^{8,10,24,18}$ but also affecting predators. ${ }^{15,17,27}$ Recently, Bairagi and Chattopadhyay ${ }^{6}$ have summarized the state-of-the-art in ecoepidemiology. Among other questions, they pointed out the interest of dealing with (nonautonomous) periodic eco-epidemiological systems, which, to the best of our knowledge, have still not been treated.

Besides, in many cases, epidemics rapidly spread. If the model couples epidemics with other process, it may be necessary to take into account the different time scales, as pointed out in Ref. 27. There, the authors suggested delayed differential equations as a possible tool to analyze this feature. Anyhow, a natural alternative consists in considering two time scale systems, as done for autonomous models in Ref. 5.

Therefore, this section fulfills that gap pointed out in Ref. 6 and contributes an alternative approach to that set out in Ref. 27.

We present a classical periodic Lotka-Volterra competition model coupled with a SIRS epidemic process affecting one of the species. Epidemic and community parameters are assumed to vary at the slow time scale (say, yearly). Considering that epidemic evolves faster than community interactions yields the following two time scales model:

$$
\left\{\begin{array}{l}
\varepsilon \frac{d S}{d t}=\gamma(t) R-\frac{\beta(t) S I}{S+I}+\varepsilon S\left(b_{1}(t)-a_{11}(t) S-a_{12}(t) w\right), \\
\varepsilon \frac{d I}{d t}=\frac{\beta(t) S I}{S+I}-\delta(t) I+\varepsilon I\left(b_{1}(t)-a_{11}(t) I-a_{12}(t) w\right), \\
\varepsilon \frac{d R}{d t}=\delta(t) I-\gamma(t) R+\varepsilon R\left(b_{1}(t)-a_{11}(t) R-a_{12}(t) w\right), \\
\frac{d w}{d t}=\varepsilon w\left(b_{2}(t)-a_{21}(t)(S+I+R)-a_{22}(t) w\right),
\end{array}\right.
$$

where all the parameters are strictly positive $T$-periodic functions of $\operatorname{class} \mathcal{C}^{2}(\mathbb{R})$. In particular, $\beta$ and $\gamma$ stand for the infection rate and the recovery rate, respectively, and parameters $a_{1 j}, b_{i}$ have the usual meaning in the classical Lotka-Volterra 
competition model. We recall that, from the point of view of applying approximate aggregation techniques, there is no loss of generality in considering the same coefficients for susceptible, infected or recovered individuals.

In order to write system (4.1) in an appropriate slow-fast form we define the slow variable $v:=S+I+R$ and change variables in (4.1) according with $(S, I, R, w) \mapsto$ $(S, I, v, w)$. The resulting system is like $(2.2)$, that is

$$
\left\{\begin{array}{l}
\varepsilon \frac{d x}{d t}=F(t, x, y)+\varepsilon \bar{S}(t, x, y), \\
\frac{d y}{d t}=S(t, x, y) .
\end{array}\right.
$$

In order to build up the reduced system, we must check Hypotheses H1 up to H4.

Hypothesis $\mathrm{H} 1$ holds because the non-negative cone of $\mathbb{R}^{4}$ is positively invariant for system (4.1).

For Hypothesis $\mathrm{H} 2$ we seek for a function $x=\phi(t, y)$ such that $0=$ $F(t, \phi(t, y), y, 0)$ (see Appendix A). Direct calculations yield the aforementioned function $\phi$ such that $\left(S_{*}, I_{*}\right)=\phi(t, v, w) \in \mathbb{R}_{+}^{2}$ (we note with a subindex $*$ the corresponding values of $S, I)$, namely:

- If $\beta(t)>\delta(t), \forall t \in\left[t_{0}, t_{0}+T\right]$ then

$$
\begin{aligned}
& S_{*}(t, v)=\frac{\gamma(t) \delta(t) v}{\gamma(t) \beta(t)+(\beta(t)-\delta(t)) \delta(t)}=\sigma_{*}(t) v, \\
& I_{*}(t, v)=\frac{\gamma(t)(\beta(t)-\delta(t)) v}{\gamma(t) \beta(t)+(\beta(t)-\delta(t)) \delta(t)}=\iota_{*}(t) v,
\end{aligned}
$$

which corresponds with the disease-endemic state. Besides, we define $R_{*}(t, v):=$ $v-\sigma_{*}(t, v)-\iota_{*}(t, v)=\rho_{*}(t) v$.

- If $\beta(t)<\delta(t), \forall t \in\left[t_{0}, t_{0}+T\right]$ then $S_{*}=v$ and $I_{*}=0$ (thus, $v-S_{*}(t)-I_{*}(t)=$ $\left.R_{*}(t)=0\right)$, which is related with the disease-free state.

Direct calculations show that $\operatorname{det}\left(F_{x}(t, \phi(t, y), y) \neq 0\right.$ for all $(t, y) \in\left[t_{0}, \infty\right) \times B_{R^{\prime}}$ in both cases, $\beta(t)>\delta(t)$ and $\beta(t)<\delta(t)$.

Regarding Hypothesis H3, it is known (see, for instance, Ref. 5) that, for each $(\alpha, \beta) \in\left[t_{0}, \infty\right) \times B_{R^{\prime}}, X=\phi(\alpha, \beta)$ is an asymptotically stable equilibrium of $\frac{d X}{d \tau}=F(\alpha, X, \beta)$ uniformly in $(\alpha, \beta) \in\left[t_{0}, \infty\right) \times B_{R^{\prime}}$. Finally, the corresponding reduced system written in terms of slow variables is

$$
\left\{\begin{array}{l}
\frac{d v}{d t}=v\left(b_{1}(t)-a_{11}^{*}(t) v-a_{12}(t) w\right), \\
\frac{d w}{d t}=w\left(b_{2}(t)-a_{21}(t) v-a_{22}(t) w\right),
\end{array}\right.
$$

where $a_{11}^{*}(t)=a_{11}(t)\left(\sigma_{*}^{2}(t)+\iota_{*}^{2}(t)+\rho_{*}^{2}(t)\right), \sigma_{*}(t)+\iota_{*}(t)+\rho_{*}(t)=1$ and $\sigma_{*}(t), \iota_{*}(t), \rho_{*}(t) \in[0,1]$. System (4.2) matches with that studied in Ref. 14 and the methodology developed herein allows us to get asymptotic information about 
the complete model (4.1) in terms the solutions of system (4.2). We obtain the following result from Ref. 14 .

Proposition 4.1. Given any $T$-periodic function $c$ we note $c^{l}:=\min \{c(t), t \in$ $[0, T]\}$ and $c^{u}:=\max \{c(t), t \in[0, T]\}$. If condition

$$
b_{1}^{l}>\frac{a_{12}^{u} b_{2}^{u}}{a_{22}^{l}}, \quad b_{2}^{l}>\frac{a_{21}^{u} b_{1}^{u}}{a_{11}^{* l}}
$$

holds, then there exists a positive periodic solution $\left(v^{*}(t), w^{*}(t)\right)$ for system $(4.2)$. In addition, if there exists $\delta>0$ such that condition

$$
a_{11}^{* l}>a_{21}^{u}+\delta, \quad a_{22}^{l}>a_{12}^{u}+\delta
$$

holds, then $\left(v^{*}(t), w^{*}(t)\right)$ is globally asymptotically stable.

Proof. For the proof, see Ref. 14.

Proposition 4.2. Consider that conditions (4.3) and (4.4) hold. Let $n(t)=$ $(S(t), I(t), R(t), w(t))$ be the solution of system (4.1) with initial values $\left(S_{0}, I_{0}\right.$, $\left.R_{0}, w_{0}\right)$ such that $\left(S_{0}, I_{0}\right)$ are in the domain of attraction of $\phi\left(t_{0}, S_{0}+I_{0}+R_{0}\right)$. Consider also $(\bar{v}(t), \bar{w}(t))$ the solution of the aggregated system (4.2) with initial values $\left(S_{0}+I_{0}+R_{0}, w_{0}\right)$. If $\beta(t)>\delta(t)$ or $\beta(t)<\delta(t)$ hold, then

$$
n(t)=\left(\sigma_{*}(t) \bar{v}(t), \iota_{*}(t) \bar{v}(t), \rho_{*}(t) \bar{v}(t), \bar{w}(t)\right)+o(1),
$$

as $\varepsilon \rightarrow 0^{+}$uniformly on any interval of the form $t_{0}<t_{1} \leq t<\infty$, where

- If $\beta(t)>\delta(t)$ then $\sigma(t), \iota(t), \rho(t)>0$.

- If $\beta(t) \leq \delta(t)$ then $\sigma(t)=1, \iota(t)=0, \rho(t)=0$.

Proof. As Hypotheses H1, H2, H3 and H4 hold, it follows from Theorem 2.1.

We point out that these coefficients involved in conditions (4.3) and (4.4) depend on the coefficients of the fast dynamics, so that these conditions may fail as a consequence of a change in the fast time scale process. A deeper analysis of this model could be done in different ways. On the one hand, investigating the relation between the existence of positive coexistence state and the stability of the semitrivial solutions of system (4.2) as we did for the predator-prey model. On the other hand, considering different population parameter values for susceptible, infected or susceptible individuals. Also different values could be considered for the competition term in the disease-free species equation. All these insights are of interest and deserve being investigated.

\section{Perspectives}

The methodology presented herein states the basis for future applications in a wide range of fields, for instance, examples in spatially distributed predator-prey models 
or eco-epidemics models have been presented in this paper. Applications in coexistence in spatially structured habitats and epidemics in implicit meta-population models are also of great interest.

In Ref. 23 the authors review the basic assumptions of the patch occupancy meta-population theory (POT), which is a key theoretical framework for studying population persistence and species coexistence in fragmented landscapes. ${ }^{21}$ This theory is based on the so-called competition-colonization trade-off; one the most common and simple theoretical explanation for the coexistence of species in mosaic habitats. According to this mechanism, poorer competitors can stably coexist with competitively superior species thanks to their greater colonizing ability (e.g. migrations). The POT focuses on whether populations occupy patches but it does not consider local dynamics. Besides, implicit to POT there is an assumption that competition operates at a much faster time scale than colonization-extinction processes. All these assumptions preclude in fact local coexistence and imply that migration cannot influence local competitive interactions. However, empirical studies have revealed a pattern that is at odds with this assumption, chiefly widespread presence of colonizers species and local coexistence. Amarasekare and Nisbet ${ }^{1}$ have shown that the mismatch between patch occupancy theory and data may arise from the separation of time scales inherent in the patch occupancy framework. Thus, it follows that the need to revisit POT underlying assumptions to incorporate a more realistic description of the temporal scales at which key ecological processes operate, as was recently done in Ref. 23 in the framework of autonomous ordinary differential equations. Subsequently, the techniques presented in this paper should allow extending these results to nonautonomous systems, which provide an even more accurate description of reality.

Epidemiology is another important field where to apply the result presented herein, as periodic patterns in epidemics are well documented. In addition, diseases in, for instance, human settlements does not evolve homogeneously. We may distinguish population clusters according with epidemic behavior, which may evolve different within the whole population, for instance, due to spatial heterogeneity of environmental conditions (different salubriousness conditions, infrastructures,...). We represent these clusters by patches which can be distant from each other and, typically, are not isolated. When individuals cannot be confined within clusters, the interest relies in understanding how epidemic behavior depends on the exchange of individuals between patches and if any class of control on population flow can contribute to handle epidemic. Thus, models should incorporate individual displacements (migrations) between patches, which can be assumed also to be periodic. The model couples processes of different nature and we distinguish between local (individual)-global (population) scales in order to tune our model. Individual displacements between patches happen at global scale while epidemic takes place at individual level, despite of how high the infection/recovery rates are. Therefore, the spread of an epidemic within each patch has small impact on the whole population and migrations is considered to be a faster process than epidemic. 


\section{Appendix A. Quasistatic-State Approximation for Nonlinear Initial Value Problems}

We summarize here the results on quasistatic-state approximation for nonlinear initial value problems, due to Hoppensteadt, ${ }^{19-21}$ that allow to extend aggregation methods (see Refs. 3 and 4) to some two time scales nonautonomous systems of ordinary differential equations, in particular to system (3.4).

We consider the initial value problem

$$
\begin{cases}\varepsilon \frac{d x}{d t}=f(t, x, y, \varepsilon), & x\left(t_{0}\right)=\xi_{0}, \\ \frac{d y}{d t}=g(t, x, y, \varepsilon), & y\left(t_{0}\right)=\eta_{0},\end{cases}
$$

where $x \in \mathbb{R}^{n}, y \in \mathbb{R}^{m}$ and $\varepsilon$ is a small positive parameter. We define the domain $\hat{\Omega}=\Omega \times\left[0, \varepsilon_{0}\right]$ where $\Omega=I \times B_{R} \times B_{R^{\prime}}, I=\left\{t: t_{0} \leq t \leq T \leq \infty\right\}, B_{R}=\{x \in$ $\left.\mathbb{R}^{n}:|x| \leq R\right\}, B_{R^{\prime}}=\left\{y \in \mathbb{R}^{m}:|y| \leq R^{\prime}\right\}, T$ and $\varepsilon_{0}$ are fixed constants. In what follows, the balls $B_{R}$ and $B_{R^{\prime}}$ can be replaced by any sets that are diffeomorphic to them.

Hypothesis H1. Functions $f$ and $g$ are $\mathcal{C}^{2}(\Omega)$ and any solution of the system (A.1) beginning in $B_{R} \times B_{R^{\prime}}$ remains there for $t_{0} \leq t \leq T$.

Setting $\varepsilon=0$ in (A.1) we obtain the so-called reduced problem:

$$
\left\{\begin{array}{l}
0=f(t, x, y, 0), \\
\frac{d y}{d t}=g(t, x, y, 0), \quad y\left(t_{0}\right)=\eta_{0} .
\end{array}\right.
$$

Hypothesis H2. There is a function $x=\Phi(t, y)$ such that $f(t, \Phi(t, y), y, 0)=0$ for $(t, y) \in I \times B_{R^{\prime}}$. Moreover $\Phi \in \mathcal{C}^{2}\left(I \times B_{R^{\prime}}\right)$ and $\operatorname{det}\left(f_{x}(t, \Phi(t, y), y, 0)\right) \neq 0$ for $(t, y) \in I \times B_{R^{\prime}}$.

Hypothesis H3. The system of equations

$$
\frac{d X}{d \tau}=f(\alpha, X, \beta, 0)
$$

has $X=\Phi(\alpha, \beta)$ as an equilibrium for each $(\alpha, \beta) \in I \times B_{R^{\prime}}$ that is asymptotically stable uniformly in the parameters $(\alpha, \beta) \in I \times B_{R^{\prime}}$, and the initial condition $\xi_{0}$ is in the domain of attraction of the equilibrium $\Phi\left(t_{0}, \eta_{0}\right)$ for system (A.3) with $\alpha=t_{0}$ and $\beta=\eta_{0}$.

Hypothesis H4. The system of equations

$$
\frac{d y_{0}}{d t}=g\left(t, \Phi\left(t, y_{0}\right), y_{0}, 0\right)
$$

has a solution for $t_{0} \leq t<\infty$, say $y^{*}(t)$, that it is uniformly asymptotically stable and $\eta_{0}$ is in the domain of attraction of $y^{*}(t)$.

Theorem A.1. Let Hypotheses $\mathrm{H} 1, \mathrm{H} 2, \mathrm{H} 3$ and $\mathrm{H} 4$ be satisfied and let $y_{0}(t)$ be the solution of (A.4) for $y_{0}\left(t_{0}\right)=\eta_{0}$. Then, for sufficiently small values of $\varepsilon$ the 
solution of problem (A.1), $(x(t), y(t))$, exists for $t_{0} \leq t<\infty$ and it satisfies

$$
x(t)=\Phi\left(t, y_{0}(t)\right)+o(1), \quad y(t)=y_{0}(t)+o(1),
$$

as $\varepsilon \rightarrow 0^{+}$uniformly on any interval of the form $t_{0}<t_{1} \leq t<\infty$.

\section{Appendix B. Lemma}

Lemma B.1. Let $b_{i j}(t)>0$, where $i, j=1,2$, be strictly positive periodic functions with period $T$. Then, the zero solution of system

$$
\left\{\begin{array}{l}
z_{1}^{\prime}=-b_{11}(t) z_{1}-b_{12}(t) z_{2} \\
z_{2}^{\prime}=b_{21}(t) z_{1}-b_{22}(t) z_{2}
\end{array}\right.
$$

is uniformly asymptotically stable.

Proof. We recall that $b_{i j}(t)$, for $i, j=1,2$, are periodic positive functions. Let us note the minimum and the maximum of each $b_{i j}(t)$, for $i, j=1,2$, as $0<b_{i j}^{L}$ and $0<b_{i j}^{M}$, respectively. Before proceeding, we recall a simple fact.

Remark B.1. Consider system

$$
Z^{\prime}(t)=B Z(t)
$$

where $B$ is given by

$$
\left(\begin{array}{cc}
-b_{11} & -b_{12} \\
b_{21} & -b_{22}
\end{array}\right)
$$

with $b_{i j}>0, i, j=1,2$ are positive real numbers. It is straightforward that the real part of the eigenvalues of (B.2) is strictly negative. Thus, the zero solution of system (B.1) is uniform asymptotically stable uniformly with respect to the initial values. We mean that, given initial values $Z_{0}$, there exist positive constants $K, \alpha \in \mathbb{R}_{+}$ such that

$$
\left\|e^{B t} Z_{0}\right\| \leq K e^{-\alpha t} \quad \forall Z_{0} ; \quad Z_{0} \leq K
$$

Getting back to our problem, let us note

$$
Y(t)=\left(\begin{array}{l}
y_{1}(t) \\
y_{2}(t)
\end{array}\right), \quad Z(t)=\left(\begin{array}{c}
z_{1}(t) \\
z_{2}(t)
\end{array}\right) .
$$

The study of the stability of the zeroth solution of system (3.15) is carried out by means of a comparison method. Namely, given a solution of system (3.15) we build up appropriate bounding linear systems with constant coefficient similar to (B.2). The solutions of these bounding systems are upper and lower bounds for the solution of system (3.15).

For this purpose, we use appropriate choices of $b_{i j}^{L}$ and $b_{i j}^{M}$ for constructing each bounding system, depending on the sign of $y_{1}$ and $y_{2}$. Without loss of generality, 
let us begin by assuming that $y_{1}\left(t_{0}\right)=y_{1}^{0}>0$ and $y_{2}\left(t_{0}\right)=y_{2}^{0}>0$. Then, in a neighborhood of $t_{0}$, it follows that

$$
\begin{gathered}
-b_{11}^{M} y_{1}(t)-b_{12}^{M} y_{2}(t) \leq y_{1}^{\prime}(t)=-b_{11}(t) y_{1}(t)-b_{12}(t) y_{2}(t) \leq-b_{11}^{L} y_{1}(t)-b_{12}^{L} y_{2}(t), \\
b_{21}^{L} y_{1}(t)-b_{22}^{M} y_{2}(t) \leq y_{2}^{\prime}(t)=b_{21}(t) y_{1}(t)-b_{22}(t) y_{2}(t) \leq b_{21}^{M} y_{1}(t)-b_{22}^{L} y_{2}(t) .
\end{gathered}
$$

Let us consider the following bounding systems:

$$
\left\{\begin{array} { l } 
{ Z ^ { \prime } ( t ) = B _ { L } Z ( t ) , } \\
{ z _ { 1 } ( t _ { 0 } ) = y _ { 1 } ^ { 0 } , } \\
{ z _ { 2 } ( t _ { 0 } ) = y _ { 2 } ^ { 0 } , }
\end{array} \quad \left\{\begin{array} { l } 
{ Y ^ { \prime } ( t ) = B ( t ) Y ( t ) , } \\
{ y _ { 1 } ( t _ { 0 } ) = y _ { 1 } ^ { 0 } , } \\
{ y _ { 2 } ( t _ { 0 } ) = y _ { 2 } ^ { 0 } , }
\end{array} \quad \left\{\begin{array}{l}
W^{\prime}(t)=B_{M} W(t), \\
w_{1}\left(t_{0}\right)=y_{1}^{0}, \\
w_{2}\left(t_{0}\right)=y_{2}^{0},
\end{array}\right.\right.\right.
$$

where $B(t)$ is that of Eq. (3.16) and $B_{L}$ and $B_{M}$ are given by

$$
B_{L}=\left(\begin{array}{cc}
-b_{11}^{M} & -b_{12}^{M} \\
b_{21}^{L} & -b_{22}^{M}
\end{array}\right), \quad B_{M}=\left(\begin{array}{cc}
-b_{11}^{L} & -b_{12}^{L} \\
b_{21}^{M} & -b_{22}^{L}
\end{array}\right),
$$

the comparison theorem yields

$$
z_{1}(t) \leq y_{1}(t) \leq w_{1}(t), \quad z_{2}(t) \leq y_{2}(t) \leq w_{2}(t), \quad t \geq t_{0},
$$

at least while $z_{1}(t), z_{2}(t), w_{1}(t), w_{2}(t)$ are kept positive (say, in an interval $I_{0}:=$ $\left[t_{0}, t^{*}\right)$ ), with $t^{*}>t_{0}$ (it may happen that $\left.t^{*}=+\infty\right)$.

Having in mind Remark B.1, it follows that $Z(t)$ and $W(t)$ decrease exponentially fast, and so does $Y(t)$ in $I_{0}$. It may happen that one of the components becomes zero after a transient time, that is, $t^{*}<+\infty$. Let us assume, without loss of generality, that $y_{1}\left(t^{*}\right)=0$ and $y_{2}\left(t^{*}\right)>0$. We recall that $\left\|Y\left(t^{*}\right)\right\|<\left\|Y\left(t_{0}\right)\right\|$. To carry on approaching the zero solution, let us replace the bounding systems (B.5) by another ones from $t^{*}$ on.

It is straightforward that there exists $\varepsilon>0$ such that $y_{1}(t)<0, y_{2}(t)>0$ and $\|Y(t)\|<\left\|Y\left(t_{0}\right)\right\|$ for all $t \in\left[t^{*}, t^{*}+\frac{\varepsilon}{2}\right]$. Thus, let us note

$$
t_{1}=t^{*}+\frac{\varepsilon}{2}, \quad y_{1}^{1}=y_{1}\left(t_{1}\right), \quad y_{2}^{1}=y_{2}\left(t_{1}\right) .
$$

Considering

$$
\left\{\begin{array} { l } 
{ Z ^ { \prime } ( t ) = B _ { L } Z ( t ) , } \\
{ z _ { 1 } ( t _ { 1 } ) = y _ { 1 } ^ { 1 } , } \\
{ z _ { 2 } ( t _ { 1 } ) = y _ { 2 } ^ { 1 } , }
\end{array} \quad \left\{\begin{array} { l } 
{ Y ^ { \prime } ( t ) = B ( t ) Y ( t ) , } \\
{ y _ { 1 } ( t _ { 1 } ) = y _ { 1 } ^ { 1 } , } \\
{ y _ { 2 } ( t _ { 1 } ) = y _ { 2 } ^ { 1 } , }
\end{array} \quad \left\{\begin{array}{l}
W^{\prime}(t)=B_{M} W(t), \\
w_{1}\left(t_{1}\right)=y_{1}^{1}, \\
w_{2}\left(t_{1}\right)=y_{2}^{1},
\end{array}\right.\right.\right.
$$

where $B(t)$ is that of Eq. (3.16) and $B_{L}$ and $B_{M}$ are now given by

$$
B_{L}=\left(\begin{array}{cc}
-b_{11}^{L} & -b_{12}^{M} \\
b_{21}^{M} & -b_{22}^{M}
\end{array}\right), \quad B_{M}=\left(\begin{array}{cc}
-b_{11}^{M} & -b_{12}^{L} \\
b_{21}^{L} & -b_{22}^{L}
\end{array}\right) .
$$

Despite of the change in the coefficients corresponding with $z_{1}$ and $w_{1}$, the leftand right-hand side systems (B.8) fit in Remark B.1. Therefore, we can repeat the previous argument, so that $Y(t)$ keeps approaching zero for $t \in\left[t^{*}, t^{*}+K\right)$ for 
certain $K>0$. Remark B.1 is general enough to hold whatever super-index $M$ or $L$ we use in the $b_{i j}$ coefficient for $i, j=1,2$.

Summing up, previous argument is independent on the sign of $y_{1}(t)$ and $y_{2}(t)$, so that it holds whatever the sign of $y_{1}(t)$ and $y_{2}(t)$ is. On the other hand, $Y(t)$ approaches uniformly exponentially fast the zero solution because of the nature of the bounding solutions.

\section{Appendix C. Proof of Proposition 3.4}

Proof of Proposition 3.4. Consider the $\varphi_{T}$ operator defined in the proof of Proposition 3.3. Let us define

$$
\begin{gathered}
F:=I-\varphi: \mathbb{R}_{+}^{2} \rightarrow \mathbb{R}_{+}^{2}, \\
(r, s) \mapsto F(r, s):=(r-n(T, r, s), s-p(T, r, s)),
\end{gathered}
$$

where $n$ and $p$ stand for the solutions of the $\left(P_{0}\right)$ problem such that $n(0)=r$ and $p(0)=s$. We already know that $\varphi_{T}$ maps $\partial R$ into $\operatorname{Int}(\mathcal{R})$, therefore $F(r, s) \neq(0,0)$ for all $(r, s) \in \partial R$. Moreover, for $\left(r_{1}, s_{1}\right) \in \operatorname{Int}(R)$ we define

$$
N(r, s, \xi):=\left(r_{1}+\xi\left[n(T, r, s)-r_{1}\right] ; s_{1}+\xi\left[p(T, r, s)-s_{1}\right]\right), \quad(r, s) \in \bar{R} .
$$

As $N(r, s, 0)=\left(r_{1}, s_{1}\right) \in \operatorname{Int}(R), N(r, s, 1)=(n(T, r, s), p(T, r, s)) \in \operatorname{Int}(R)$ and $R$ is convex, it is clear that

$$
(r, s, \xi)-N(r, s, \xi) \neq(0,0) \quad \forall(r, s) \in \partial R, \quad \xi \in[0,1] .
$$

Therefore, $N$ establishes an admissible homotopy between

$$
F(r, s)=(r, s)-N(r, s, 0) \quad \text { and } \quad\left(r-r_{1}, s-s_{1}\right)=(r, s)-N(r, s, 1)
$$

and

$$
d\left[F, R_{2}, 0\right]=d\left[\left(r-r_{1}, s-s_{1}\right), R_{2}, 0\right]=1 .
$$

We must show that $\left|J F\left(P_{0}, p_{0}\right)\right|>0$ for each positive $T$-periodic solution $\left(n_{0}, p_{0}\right)$ :

$$
\left|J F\left(P_{0}, p_{0}\right)\right|=\left|\begin{array}{cc}
1-n_{n}^{\prime}\left(T, n_{0}, p_{0}\right) & -n_{p}^{\prime}\left(T, n_{0}, p_{0}\right) \\
-p_{n}^{\prime}\left(T, n_{0}, p_{0}\right) & 1-p_{p}^{\prime}\left(T, n_{0}, p_{0}\right)
\end{array}\right|
$$

and the eigenvalues of the matrix

$$
\left(\begin{array}{cc}
n_{n}^{\prime}\left(T, n_{0}, p_{0}\right) & n_{p}^{\prime}\left(T, n_{0}, p_{0}\right) \\
p_{n}^{\prime}\left(T, n_{0}, p_{0}\right) & p_{p}^{\prime}\left(T, n_{0}, p_{0}\right)
\end{array}\right)
$$

are the characteristic multipliers $\lambda_{1}$ and $\lambda_{2}$ of the linearization of the reduced system (3.6) at the positive periodic solution. We already know that system (3.13) has no periodic solutions different from the zero solution. Then, neither 1 nor -1 
is characteristic multiplier of system (3.13). In fact, such a solution is attractive, which implies that $\left|\lambda_{i}\right|<1$ for $i=1,2$. Now, it is clear that

$$
\left|J F\left(n_{0}, p_{0}\right)\right|=\left(1-\lambda_{1}\right)\left(1-\lambda_{2}\right)>0,
$$

which concludes with the proof of the uniqueness.

\section{Acknowledgments}

The authors are partially supported by projects MTM2011-24321 and MTM201125238. M.M. thanks Professor Poggiale for his hospitality during his visits at the Laboratoire de Microbiologie, Geochimie et dEcologie Marines, at Marseille.

\section{References}

1. P. Amarasekare and R. M. Nisbet, Spatial heterogeneity, source-sink dynamics, and the local coexistence of competing species, Amer. Nat. 158 (2001) 572-584.

2. R. M. Anderson, R. M. May, K. Joysey, D. Mollison, G. R. Conway, R. Cartwell, H. V. Thompson and B. Dixon, The invasion, persistence and spread of infectious diseases within animal and plant communities [and discussion], Phil. Trans. Roy. Soc. B 314 (1986) 533-570.

3. P. Auger, R. Bravo de la Parra, J. C. Poggiale, E. Sánchez and T. Nguyen Huu, Aggregation of variables and applications to population dynamics, in Structured Population Models in Biology and Epidemiology, eds. P. Magal and S. Ruan, Lecture Notes in Mathematics, Vol. 1936 (Springer, 2008), pp. 209-263.

4. P. Auger, R. Bravo de la Parra, J. C. Poggiale, E. Sánchez and L. Sanz, Aggregation methods in dynamical systems and applications in population and community dynamics, Phys. Life Rev. 5 (2008) 79-105.

5. P. Auger, R. Mchich, T. Chowdhury, G. Sallet, M. Tchuente and J. Chattopadhyay, Effects of a disease affecting a predator on the dynamics of a predator-prey system, J. Theor. Biol. 258 (2009) 344-351.

6. N. Bairagi and J. Chattopadhyay, The evolution on eco-epidemiological systems theory and evidence, J. Phys.: Conf. Ser. 96 (2008) 1-8.

7. J. R. Beddington, Mutual interference between parasites or predators and its effect on searching efficiency, J. An. Ecol. 44 (1975) 331-340.

8. E. Beltrami and T. O. Carroll, Modeling the role of viral disease in recurrent phytoplankton blooms. J. Math. Biol. 32 (1994) 857-863.

9. L. E. J. Brouwer, Ueber eineindeutige, stetige Transformationen von Flächen in sich, Math. Ann. 69 (1910) 176-180.

10. J. Chattopadhyay and O. Arino, A predator-prey model with disease in the prey, Nonlinear Anal. 36 (1999) 747-766.

11. D. L. DeAngelis, R. A. Goldstein and R. V. O'Neill, A model for tropic interaction, Ecol. 56 (1975) 881-892.

12. M. Farkas, Periodic Motions (Springer, 1994).

13. N. Fenichel, Persistence and smoothness of invariant manifolds for flows, Ind. Univ. Math. J. 21 (1971) 193-226.

14. K. Gopalsamy, Global asymptotic stability in a periodic Lotka-Volterra system, J. Austral. Math. Soc. Ser. B 27 (1985) 66-72.

15. L. Han, Z. Ma and H. W. Hethcote, Four predator-prey models with infectious diseases, Math. Comput. Model. 34 (2001) 849-858. 
16. I. Hanski, Metapopulation Ecology (Oxford Univ. Press, 1999).

17. M. Haque and E. Venturino, An eco epidemiological model with disease in predator: The ratio-dependent case, Math. Models Methods Appl. Sci. 30 (2007) 1791-1809.

18. H. W. Hethcote, W. Wang, L. Han and Z. Ma, A predator-prey model with infected prey, Theor. Pop. Biol. 66 (2004) 259-268.

19. F. Hoppensteadt, Singular perturbations on the infinite interval, Trans. Amer. Math. Soc. 123 (1966) 521-535.

20. F. Hoppensteadt. Analysis and Simulation of Chaotic Systems (Springer, 1993).

21. F. Hoppensteadt, Quasi-Static State Analysis of Differential, Difference, Integral, and Gradient Systems (Courant Institute of Mathematical Sciences, New York Univ., 2010).

22. H. Lischke, T. J. Löffler, P. E. Thornton and N. E. Zimmermann, Model up-scaling in landscape research, in A Changing World. Challenges for Landscape Research, eds. F. Kienast, O. Wildi and S. Ghosh, Vol. 8 (Springer, 2007), pp. 249-272.

23. D. Nguyen Ngoc, R. Bravo de la Parra, M. A. Zavala and P. Auger, Competition and species coexistence in a meta-population model: Can fast asymmetric migration reverse the outcome of competition in a homogeneous environment? J. Theor. Biol. 266 (2010) 256-263.

24. C. Packer, R. Holt, P. Hudson, K. Lafferty and A. Dobson, Keeping the herds healthy and alert: Implications of predator control for infectious disease, Ecol. Lett. 6 (2003) 797-802.

25. J. C. Poggiale and P. Auger, Fast oscillating migrations in a predator-prey model, Math. Models Methods Appl. Sci. 6 (1996) 217-226.

26. D. C. Schneider, The rise of the concept of scale in ecology, Bioscience 51 (2001) $545-553$.

27. E. Venturino, Epidemics in predator-prey models: Disease in the predators, IMA J. Math. Appl. Med. Biol. 19 (2002) 185-205.

28. F. Verhulst, Methods and Applications of Singular Perturbations, Boundary Layers and Multiple Timescale Dynamics (Springer, 2005).

29. F. Verhulst, Singular perturbation methods for slow-fast dynamics, Nonlinear Dynam. 50 (2007) 747-753.

30. C. Walters and J. F Kitchell, Cultivation/depensation effects of juvenile survival and recruitment: Implications for the theory of fishing, Canad. J. Fish. Aqu. Sci. 58 (2001) $39-50$. 